\title{
Michel Heichette, Société, sociabilité, justice. Sablé et son pays au XVIIIe siècle
}

\section{Dorothée Perrenes}

\section{Q OpenEdition \\ $\checkmark$ Journals}

Édition électronique

URL : https://journals.openedition.org/abpo/874

DOI : $10.4000 / a b p o .874$

ISBN : 978-2-7535-1502-4

ISSN : $2108-6443$

\section{Éditeur}

Presses universitaires de Rennes

\section{Édition imprimée}

Date de publication : 30 juin 2006

Pagination : 207-210

ISBN : 978-2-7535-0331-1

ISSN : 0399-0826

\section{Référence électronique}

Dorothée Perrenes, "Michel Heichette, Société, sociabilité, justice. Sablé et son pays au XVIIle siècle »,

Annales de Bretagne et des Pays de l'Ouest [En ligne], 113-2 | 2006, mis en ligne le 30 juin 2008, consulté le 22 juillet 2022. URL : http://journals.openedition.org/abpo/874; DOI : https://doi.org/10.4000/abpo. 874 
des séductions " (p. 191). Cela est le début d'une série de tensions entre les proDupont de La Motte et les pro-abbé Macé. C'est dans ce contexte que le $1^{\text {er }}$ février 1776, la nouvelle tombe : le roi supprime l'École militaire et le collège préparatoire. Les dernières pages du journal nous montrent l'investissement de l'inspecteur dans les derniers moments d'existence du collège. Il note le départ du collège de "presque tous les sous-maîtres " le vendredi 10 mai 1776 ainsi que l'absence complète d'obéissance des élèves qui " savent qu'on n'est plus rien " (p. 382). Il ne cache pas sa tristesse le 3 août 1776 : « tous ses adieux ajoutent des peines et des soins à ceux que j'ai déjà ". Le mardi 24 septembre 1776 marque la fin du collège avec l'arrivée des Doctrinaires.

Dans une troisième et dernière partie, Didier Boisson traite de ses sources sur le Collège de La Flèche. Pour fonder ses riches commentaires dans l'introduction et ses nombreuses notes de bas de page, l'auteur a eu recours aux archives. Les archives départementales de la Sarthe tout d'abord où il s'est intéressé aux jugements et registres d'audience de la sénéchaussée et du présidial de La Flèche (série B) et à la série D contenant par exemple les lettres patentes du Roi ou les délibérations de l'administration du collège. Il a également étudié les archives notariales (série $4 \mathrm{E}$ ) pour vérifier dans l'inventaire de l'appartement de Dupont de La Motte la présence de biens mentionnés dans le journal. On peut se reporter à la page 220 pour y trouver un exemple : Dupont de La Motte a reçu une " pendule à secondes " que Didier Boisson a repérée dans l'inventaire de l'inspecteur. Ensuite, les Archives du Service historique de l'armée de Terre (SHAT) ont permis à l'auteur d'avoir des renseignements sur certains élèves boursiers et sur diverses affaires notées dans le journal. Enfin, aux Archives Nationales, les sources sur l'École militaire de Paris ont fourni quelques références relatives au collège de La Flèche. La bibliothèque du Prytanée national militaire de La Flèche conserve les trois copies du Journal de Stanislas de La Motte. Au département des manuscrits de la Bibliothèque Nationale, l'auteur a consulté les dossiers d'élèves. La Bibliothèque municipale d'Angers a permis l'étude de divers actes royaux: des lettres patentes, des ordonnances du roi, des arrêts du conseil d'État. Les pages 399 à 426 fournissent deux index clairs et complets des noms propres et des noms de lieux.

Il ne faut pas occulter l'aspect subjectif d'un journal : il n'est pas facile de déceler les parts de vérité dans le récit des événements écrit par Dupont de La Motte. Son statut d'inspecteur peut nous amener à lui reprocher, nous lecteurs du XXI ${ }^{e}$ siècle, son excès de zèle. Cependant, cet ouvrage est un instrument de travail nécessaire aux chercheurs de l'enseignement pour aborder les méthodes d'éducation de la fin du XVIII et à ceux qui s'intéressent tout particulièrement au fameux collège de La Flèche pendant cette période.

Nadège CocHAIN

Heichette, Michel, Société, sociabilité, justice. Sablé et son pays au xvIII siècle, Rennes, PUR, coll. « Histoire ", 2005, 324 p.

Nous pouvons dire que les archives judiciaires sont au cœur de cette étude mais le but de Michel Heichette n'est pas d'écrire une histoire de la criminalité. Ce travail s'inscrit plutôt dans le champ de l'histoire sociale. Il s'intéresse aux formes de la sociabilité, aux comportements des individus en société et à leurs façons de vivre ensemble. Dans cet ouvrage, l'auteur commence par la présen- 
tation des sources utilisées : les archives judiciaires, puis à une présentation du " terrain de l'enquête ": Sablé et son pays au XvIII" siècle. Il veut savoir comment les hommes ont exploité ces lieux. Par la suite, il traite des façons de vivre ensemble, de la place de la famille dans les relations sociales. Michel Heichette évoque également les moments de détente présents dans le jeu social des gens de Sablé. Nous avons ici une véritable analyse des mises en scènes de la vie quotidienne. Enfin, l'auteur termine son étude en évoquant d'une part la place primordiale que tiennent l'honneur et la réputation dans la vie communautaire et d'autre part les systèmes de valeur villageois, à savoir, le respect de la propriété, de l'autorité et du sacré ainsi que le modèle de l'honnête homme et de la femme d'honneur.

On voit que Michel Heichette s'est tout d'abord intéressé à la source et aux protagonistes du jeu social. L'archive judiciaire se trouve au cœur de l'enquête et on s'aperçoit que les fonds judiciaires saboliens sont abondants. Le corpus documentaire est dense et varié. Les dépositions de témoins et les interrogatoires sont des documents très riches. On s'aperçoit que la justice traite d'abord les problèmes de la vie quotidienne, c'est une justice de proximité. Les archives judiciaires permettent d'observer une grande diversité de situation et d'acteurs en montrant les formes diverses de sociabilité. Ensuite, l'auteur souhaite cerner le lieu de l'enquête dans lequel le jeu social se met en place. Le territoire choisi par Michel Heichette comme terrain d'observation nous montre un monde plutôt ordinaire. Le pays de Sablé paraît représentatif des espaces de l'Ouest bocager et se situe entre le Maine et les terres angevines. Il se compose d'une vingtaine de paroisses autour d'un petit centre urbain qui concentre les pouvoirs et les activités. Ces activités sont diverses : travail agricole (production céréalière et élevage), artisanat traditionnel, activités d'échange dans les marchés et les foires du centre sabolien... Le tissu social sabolien se compose de bourgeois et d'officiers, de petites gens (anonymes, commerçants...), de marchands fermiers et de gens de campagne.

Les relations familiales sont primordiales à Sablé, la famille représente la valeur essentielle. À travers la lecture des archives judiciaires, Michel Heichette a pu constater que la famille, fondée sur le mariage, constitue un espace de solidarité, de protection, de refuge et un espace de socialisation. Il s'agit d'un mode de relation privilégié au cœur de la sociabilité villageoise et un fondement de la société d'Ancien Régime. Il apparaît que l'enfant est au cœur des préoccupations et le devoir des parents est de pourvoir aux besoins essentiels de leur progéniture. Les relations parents-enfants se fondent sur la protection, l'entraide et le respect. En ce qui concerne le couple, on observe une certaine répartition des rôles et des responsabilités. On peut distinguer des domaines réservés. La maison et son domaine proche constituent l'espace féminin. L'espace masculin se trouve hors du foyer et se compose du chantier, des champs, du marché, du cabaret, c'est-à-dire un champ d'action tourné vers l'extérieur. Mais on peut observer une certaine complémentarité dans le couple. Les champs d'activité ne sont pas clos et les deux époux se partagent l'espace et les responsabilités. À Sablé, il apparaît que la femme bénéficie d'une autonomie importante et d'un pouvoir de décision, elle n'est cependant pas libre au sens juridique du terme. Les femmes constituent un des piliers essentiels de la société en régnant sur la sphère du privé.

Une part importante est laissée aux moments de détente dans la vie des habitants de Sablé au XvIII ${ }^{\mathrm{e}}$ siècle. Ce sont des temps forts de l'intégration sociale. Les dimanches et les fêtes religieuses constituent des moments de rupture avec les jours de labeur. Se rendre au cabaret est une pratique courante et relève du 
domaine masculin, c'est un point central. Il s'agit d'un lieu où l'on peut partager des boissons et de la nourriture. Le cabaret est également un lieu de paroles et de convivialité. Il peut, si on en use mal, devenir un lieu de perdition. Michel Heichette distingue dans les sources qu'il utilise d'autres formes de détente. On peut ainsi parler de promenade en groupe, de visites entre voisins ou entre amis. Il existe des moments privilégiés comme les fêtes familiales. Les marchés et les foires sont des lieux de rencontres et d'échange verbal très ouvert sociologiquement. Ces lieux rassemblent un monde diversifié avec des individus de sexe, âge et statut social différents. La présente étude insiste sur le fait que la société veut se préserver de maux qui peuvent attenter à l'ordre familial, social et moral. L'importance ici est d'étudier les attitudes de la société face aux transgressions des normes établies. On observe ainsi des déviances et des excès qui sont souvent ceux d'une jeunesse masculine à qui l'on reproche de trop se divertir. Par conséquent, il existe un risque pour les filles : succomber aux pièges de la séduction. Ces comportements sont dénoncés mais la société et les familles savent s'accommoder, en recevant par exemple, des dommages et intérêts.

Michel Heichette a pu observer que les pièces de procédure concernant Sablé sont imprégnées d'un discours sur l'honneur. Le XvIII siècle se caractérise donc par le fait que la société villageoise est une société où l'honneur est une valeur fondamentale du jeu social. L'individu tient par-dessus tout à revendiquer sa réputation et sa dignité. Une certaine violence est constatée dans les sources. Elle n'est pas prioritairement le fait de délinquants mais plutôt d'un monde ordinaire. Son origine principale est la défense de l'honneur et il faut préserver sa réputation par tous les moyens. Il ressort de l'étude des sources judiciaires que les injures et les diffamations à caractère sexuel sont les plus nombreuses et les plus infamantes. Elles visent d'abord les femmes et font très souvent allusions à l'infidélité des épouses. L'insulte n'est pas le fait d'une catégorie sociale particulière mais elle est assez courante chez les hommes. La plainte naît de la violence subie, aussi, déposer plainte contre autrui revient à revendiquer un démenti nécessaire. L'honneur et la réputation sont "le bien le plus précieux, préférable à toutes les richesses du monde ». Il faut ainsi rétablir son caractère d'honnête homme aux yeux de la communauté si celui-ci est menacé. Il faut savoir que l'écho public fait la réputation dans la société villageoise et que le regard d'autrui prime sur le reste. L'honneur et la réputation constituent bien les moteurs de la relation sociale. Ces deux concepts représentent les fondements des sociétés villageoises d'Ancien Régime.

Dans cette étude, Michel Heichette s'intéresse à la définition de l'honnête homme. Il souhaite cerner le sens et la place du concept d'honnêteté dans les relations au sein de la communauté. Il s'est également intéressé aux conduites sociales à l'égard de la propriété et des biens. On observe un fort sentiment d'appropriation : l'individu à besoin de recourir au droit, au contrat et à l'usage. En ce qui concerne la propriété, toute violation est vue comme une atteinte à la dignité, ainsi sauver ce que l'on possède revient à défendre son honneur. Les attitudes de la société face aux vols constituent un point intéressent. Ce type de délit se trouve au second rang des plaintes reçues par le tribunal de Sablé. Il en émerge un ensemble de valeurs dont, l'importance accordée aux biens et à leur sécurité, l'affirmation du droit de défendre son patrimoine et le respect de ce que chacun possède. Michel Heichette montre que l'opinion commune est confiante vis-à-vis de la justice de proximité. Il y a donc un certain respect des rôles sociaux. Cette justice reconnaît un droit, celui de retrouver un honneur. Elle constitue une protection contre la violence, contre l'atteinte à l'ordre public. Mais pour certains individus la justice est crainte ou encore sujette à des 
défiances et des provocations. La source judiciaire permet aussi de se rendre compte des attitudes face à la religion. Il y a un véritable respect de Dieu et des lieux de culte. L'église constitue un autre " théâtre du jeu social " et il faut savoir que le respect du sacré est une qualité de l'honnête homme. La conscience des rôles est forte à tous les niveaux de l'échelle sociale. On a besoin d'une dignité reconnue, de considération et de respect. L'honnêteté est un idéal de vertu à la fois sociale et morale et trouve ses fondements dans un ensemble de codes sociaux et de devoirs moraux à respecter. L'honnête homme doit refuser la violence et contribuer à l'harmonie de la société. La femme doit respecter les vertus conjugales et maternelles : la fidélité, dont dépend l'honneur familial et, le mariage. Il existe donc un code de l'honneur reconnu par l'opinion commune. Il répond aux préoccupations des hommes de pouvoir, de justice et d'église.

À travers cet ouvrage, Michel Heichette a cherché à comprendre les sensibilités collectives et les comportements des gens de l'Ouest. Il a retracé leur histoire en s'imprégnant de la banalité de leur vie mais en l'insérant dans la dynamique du jeu social.

Dorothée PERENNES

WACHE, Brigitte (dir.), Militants catholiques de l'Ouest. De l'action religieuse aux nouveaux militantismes (XIX'-XXe siècle), Rennes, PUR, coll. " Histoire ", 2004, $250 \mathrm{p}$.

Les actes de ce colloque très important, qui s'est tenu à l'Université du Maine, les 24 et 25 janvier 2003, sous la direction de Brigitte Waché, Doyen de la Faculté des Lettres, Langues et Sciences humaines, représentent non seulement une œuvre originale, en matière de recherche, mais aussi une contribution forte à la connaissance de l'Ouest.

Sa nouveauté réside autant dans ses résultats que dans sa démarche. En effet, il comporte des communications faites par des chercheurs universitaires comme toutes les journées d'études, mais il intègre des témoignages de militants qui ont été les acteurs de ces mouvements catholiques. Parmi eux, René Rémond, qui a assisté à l'ensemble du colloque et qui a tiré magistralement les conclusions d'un débat extrêmement riche. Enfin, le colloque a été pluridisciplinaire, regroupant des historiens, des géographes, des sociologues et des politologues.

L'objectif fixé par Brigitte Waché était "d'ébaucher un bilan des travaux réalisés sur les militants catholiques de l'Ouest français et de poser des jalons pour de futures recherches ". L'idée de "militants " renvoie à une "action menée avec conviction pour changer une situation ou promouvoir une évolution". Cette Action catholique, elle-même conduite par des laïcs, qui veulent être une élite catholique, agissant en groupe, sous diverses formes, en liaison avec le clergé, renvoie à une tradition, mais ne remonte guère avant le $\mathrm{xx}^{\mathrm{e}}$ siècle. Elle acquiert, certes, une connotation institutionnelle sous le pontificat de Pie XI (1921-1939), même si l'expression apparaissait déjà sous la plume de Lamennais vers 1830 . L'Action Catholique de la Jeunesse Française, premier mouvement de jeunesse, est née en 1886. La formule s'impose dans les années 1920, quand la JOC (Jeunesse Ouvrière Catholique), née en dehors de ces structures, va imposer son expérience spécifique. Lors de ce colloque, la JOC et la JAC se taillent une place de choix, mais Brigitte Waché regrette l'absence du monde scolaire et étu- 